\title{
Adomian Decomposition Method for Solving the Nonlinear Heat Equation
}

\author{
Shawqi Malek Alhaddad* \\ *(Public Authority for Applied Education and Training (PAAET), Kuwait, Email: sm_8449@hotmail.com)
}

\begin{abstract}
This paper studies the application of the Adomian Decomposition Method to find the exact and approximate solutions of the heat equation with power nonlinearity. First, the relevant literature is studied in understanding the importance and extent of applicability of the method in the applied science. The literature review has been incorporated in the introduction of the paper. The rest of the paper is divided in three further sections. The first part Adomian Decomposition Method provides a step-by-step guide of applying the method on any heat equation with nonlinearity. The second section is labeled as Applications. It considers two examples from the previous works of Pumak (2005) and Hetmaniok et al. (2010) to find the exact and approximate solutions of the equations respectively.
\end{abstract}

\section{INTRODUCTION}

Contemporary mathematics faces major challenges in solving problems of various differential equations such as Cauchy problems. Various methods such as differential transform method (Smarda\&Dibl'ik, 2013), Taylor collection method (Sezer\&Akyuz-Dascioglu, 2006), variational iterative method (Chen \& Wang, 2010), homotopy perturbation method (Shakeri\&Dehghan, 1994) and homtopy analysis methods (Wang, 2010) have been considered by the researchers over the past two decades. Adomian's decomposition method is of great interest to applied sciences today. It is necessary for the solution of many different problems such as partial differential equations (Adomian, 1994), algebraic equations (Al-Hayani\&Casasus, 2005), and boundary value problems (Wazwaz, 2005). It is a great method to accurately compute a rapidly convergent series solution. The main advantage of the method lies in its direct applicability to homogeneous or inhomogeneous, linear or nonlinear, integral and differential equations, and with variable or constant coefficients. Also, it maintains the high accuracy of the numerical solutions and reduces the great computational work simultaneously. The most recent application of the Adomian Decomposition Method lies in solving the heat equations with power nonlinearity (Akram\& Pasha, 2005).

\section{ADOMIAN DECOMPOSITION METHOD}

George Adomian proposed the Decomposition Method in the 1980s to solve nonlinear function equations (Cherruault, 1995). This method has been read and applied ever since (Babolian\&Biazar, 2002; Lesnic, 2002). This method is based on separating the linear and nonlinear portions of any given equation and then inverting the linear operator of the equation and applying the inverted operator to the equation. The nonlinear portion of any given equation is decomposed in various Adomian polynomials which are used to determine the recursive relationship among the terms of the series. Adomian Decomposition Method produces the convergent series solution (Hashim, 2006). Many researchers have been investing the issue of convergence (Cherruault et al., 1995; Babolian\&Biazar, 2002; Lesnic, 2002). The definition from which the order of convergence for the method could be determined was provided by Babolian\&Biazar (2002). It has also been found that the series produced by the decomposition method is absolutely convergent as well as uniformly convergent (Cherruault et al., 1995) where a high degree of convergence is desirable. It is for this reason that the series converges more rapidly. The researchers have also pointed out various advantages and disadvantages of the decomposition method. The method significantly reduces the computational work (Bulut, 2004). The method can widely handle quite general nonlinearities (Wang, 2004). It enables the researchers to develop an analytical, reliable solution of the nonlinear problems without linearization. Alongside, the method also suffers from certain disadvantages as well. First of all, the major concern lies in the region and rate of convergence of the series produced by the Adomian Decomposition Method. Also, it has very slow convergence rate in the wider regions (Jiao et al., 2002). However, a further investigation in this particular area is desirable.Nonetheless, the 
Adomian Decomposition Method is one of the finest solutions to the nonlinearities today. The advantages of the method provide a strong base of wide-ranging applicability of the decomposition solution in fields like engineering, chemistry, biology, and physics (Adomian, 1995, 1996; Babolian\&Biazar, 2002; Biazar, 2006). In fact, the method has proven to be highly applicable to such diverse areas as nonlinear optics, particle transport, mass and/or heat transfer, chaos theory and the fermentations theory (Hashim et al., 2006). The most recent application of the decomposition method lies in finding the solution of the nonlinear heat equations which are one of the most important phenomena in engineering, physics, and mathematics. Here is an example of how the decomposition method can be used to solve a simple heat equation with a power nonlinearity:

$u_{t}(x, t)=u_{x x}+u^{m}$

subject to the initial condition

$u(x, 0)=f(x)$

Approximating by an operator $L_{t} u(x, t)=L_{x x} u+u^{m}(3)$

Taking the inverse operator of the operator $L_{t}$ exists and it defined as

$$
L_{t}^{-1}(.)=\int_{0}^{t}(.) d t(4)
$$

Thus, applying the inverse operator $L_{t}^{-1}$ to equation 1 yields

$L_{t}^{-1} L_{t} u(x, t)=L_{t}^{-1} L_{x x} u+\varepsilon L_{t}^{-1} u^{m}(5)$

$u(x, t)=u(x, 0)+L_{t}^{-1} L_{x x} u+\varepsilon L_{t}^{-1} u^{m}$.

Presenting the Adomian Decomposition Method solution as under

$u(x, t)=\sum_{n=0}^{\infty} u_{n}(x, t)(7)$

Decomposing the nonlinear portion of equation 1 in Adomian polynomials

$A_{n}=\frac{1}{n !} \frac{d^{n}}{d \lambda^{n}}\left[N\left(\sum_{n=0}^{\infty} \lambda^{n} u_{n}\right)\right]_{\lambda=0} \quad, n \geq 0$

thus,

$N u(x, t)=\sum_{n=0}^{\infty} A_{n}$

Substituting equation 7 in equation 9

$u(x, t)=u(x, 0)+L_{t}^{-1} L_{x x} \sum_{n=0}^{\infty} u_{n}(x, t)+L_{t}^{-1} \sum_{n=0}^{\infty} A_{n}$.

Recurrent relation from equation 10 can be defined as

$u_{0}(x, 0)=f(x)$

$u_{n+1}(x, t)=L_{t}^{-1} L_{x x} u_{n}(x, t)+\varepsilon L_{t}^{-1} A_{n}$ for $n=0,1,2, \ldots$ form which

$$
\begin{aligned}
& u_{1}(x, t)=L_{t}^{-1} L_{x x} u_{0}+L_{t}^{-1} A_{0} \\
& u_{2}(x, t)=L_{t}^{-1} L_{x x} u_{1}+L_{t}^{-1} A_{1} \\
& u_{a}(x, t)=L_{t}^{-1} L_{x x} u_{2}+L_{t}^{-1} A_{2} \\
& \vdots \\
& u_{n}(x, t)=L_{t}^{-1} L_{x x} u_{n-1}+L_{t}^{-1} A_{n-1}
\end{aligned}
$$

We can estimate the approximate solution $\phi_{Y}$ by using the $\gamma$-term approximation. That is ,

$\phi_{Y}=\sum_{n=0}^{\gamma-1} u_{n}(x, t)$

Equation 7 and equation 11 make it clear that $u(x, t)=\lim _{\gamma \rightarrow \mathrm{s}} \phi_{\gamma}(x, t)$

Finally, we want to apply the Adomian Decomposition Method to two examples and compare the exact solution and approximate solution. The importance of arriving at approximate (Dehghan, 2004; Grzymkowski\&Słota, 2005; Hetmaniok et al., 2010) or exact (Lesnic, 2002; Pamuk\&Pamuk, 2014) solution is explained by the efficiency of these problems. Pumak (2005) utilised the Adomian Decomposition Method to find the exact location of a heat equation with both linear and nonlinear powers in his paper titled "An Application for Linear and Nonlinear Heat Equations by Adomian's Decomposition Method." For the purpose of this paper, I am considering the nonlinearity only. Here is the heat equation Pumak considered in the paper;

$$
u_{t}(x, t)=u_{x x}-2 u^{a}
$$

\section{Applications}

Consider the nonlinear heat equation and subject to initial condition

$$
\begin{aligned}
& u_{t}(x, t)=u_{x x}-2 u^{2}(12) \\
& u(x, 0)=\frac{1+2 x}{x^{2}+x+1}(13)
\end{aligned}
$$

Where for the exact solution of equation 12 as

$$
u(x, t)=\frac{1+2 x}{x^{2}+x+6 t+1}
$$

In an operator form, equation 12 becomes,

$u(x, t)=u(x, 0)+L_{t}^{-1} L_{x x} u-2 L_{t}^{-1} u^{a}(15)$

the Adomian Polynomials are

$A_{0}=-2 u_{0}^{3}$

(9)

$A_{1}=-6 u_{0}^{2} u$

$A_{2}=-6\left(u_{0} u_{1}^{2}+u_{0}^{2} u_{2}\right)$

And so on. Thus,

$$
\begin{aligned}
& u_{0}=\frac{1+2 x}{x^{2}+x+1} \\
& u_{1}=L_{t}^{-1} L_{x x}\left(u_{0}\right)-2 L_{t}^{-1}\left(u_{0}^{\mathrm{a}}\right)=\frac{-6(1+2 x)}{\left(x^{2}+x+1\right)^{2}} t \\
& u_{2}=L_{t}^{-1} L_{x x}\left(u_{1}\right)-6 L_{t}^{-1}\left(u_{0}^{2} u_{1}\right)=\frac{36(1+2 x)}{\left(x^{2}+x+1\right)^{2}} t^{2}
\end{aligned}
$$


$\left.u_{0}=L^{-1} u^{-1}\left(u_{2}^{2}\right)-6 u^{-1}+u^{2} u_{0}\right)=\frac{-216(1+2 x)}{\left(x^{2}+x+1\right)} t^{[8] . \quad J . ~ B i a z a r . ~(2006) ~ S o l u t i o n ~ o f ~ t h e ~ e p i d e m i c ~}$ model by Adomian decomposition method. Applied Mathematics and Computation, 173:1101-1106.

and so on.

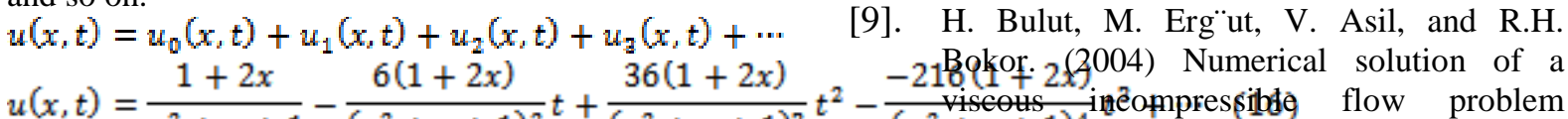

$u(x, t)=\frac{1}{x^{2}+x+1}-\frac{\left.x^{2}+x+1\right)^{2}}{\left(x^{2}+x+1\right)^{3}} t^{2}-\frac{v i s c o u s}{\left(x^{2} \text { through } 1^{4} \text { an opopressibde flow problem }\right.}$

So the equation 16 is almost equal

$u(x, t) \approx \frac{1+2 x}{x^{2}+x+6 t+1}$

It is the solution of the heat equation 12 by using ADM.

\section{CONCLUSION}

This paper proposes the application of the Adomian Decomposition Method for solving the nonlinear heat equations. It is a semi analytical method and it is the best method to solve the ordinary and partial equation with both linear and nonlinear powers. Examples from the previous works are used. It is concluded that Adomian Decomposition Method provides both exact and approximate numerical solutions for the heat equations with nonlinear power in comparison to other solutions such as antireductiori method (Fushchych\& Zhdanov, 1994) and Lie symmetry reduction method (Euler \& Euler, 1997).

\section{REFERENCES}

[1]. M. Wazwaz, (2005) Adomian decomposition method for a reliable treatment of the Bratu-type eqautions, Appl. Math. Comput. 166, 652-663.

[2]. D. Lesnic. (2002) Convergence of Adomian's decomposition method: periodic temperatures. Computers and Mathematics with Applications, 44:13-24

[3]. E. Babolian and J. Biazar. (2002) On the order of convergence of Adomian method. Applied Mathematics and Computation, 130:383-387.

[4]. F. Shakeri, M. Dehghan, (2008) Solution of delay differential equations via a homotopy perturbation method, Mathematical and Computer Modelling, 48, 486-498.

[5]. G. Adomian, Solving Frontier Problems of Physics: The Decomposition Method, Kluwer Academic Publishers, Dordrecht, 1994.

[6]. G. Adomian. (1995) Solving the mathematical models of neurosciences and medicine. Mathematics and Computers in Simulation, 40:107-114.

[7]. G. Adomian. (1996) The KadomtsevPetviashvili Equation. Applied Mathematics and Computation, 76:95-97. decomposition method. Applied

Mathematics and Computation, 153:733741.

[10]. Hetmaniok. E., Slota, D., Witula R., Zielonka A., (2010). Comparison of the Adomian decomposition method and the variational iteration method in solving the moving boundary problem. nstitute of Mathematics, Silesian University of Technology, Kaszubska 23, 44-100 Gliwice, Poland

[11]. Hashim. (2006) Comments on "a new algorithm for solving classical Blasius equation" by L. Wang. Applied Mathematics and Computation, 176(2):700-703.

[12]. Hashim, M.S.M. Noorani, and M.R. Said Al-Hadidi. (2006) Solving the generalized BurgersHuxley equation using the Adomian decomposition method. Mathematical and Computer Modelling, 43(11-12):1404-1411.

[13]. L. Wang. (2004) A new algorithm for solving classical Blasius equation. Applied Mathematics and Computation, 157:1-9.

[14]. M. Akram and M. A. Pasha, (2005) "A numerical method for the heat equation with a nonlocal boundary condition," Intern. Jour. of Information and systems sciences, vol. 1 $\mathrm{n}^{\circ} 2$, pp.162- 171.

[15]. M. Euler, N. Euler, (1997). Symmetries for a class of explicitly space and time dependent $(1+1)$ - dimensional wave equations, Sym. Nonlinear Math. Phys. 1, 70-78.

[16]. M. Dehghan, (2004). Application of the Adomian decomposition method for twodimensional parabolic equation subject to nonstandard boundary specifications. Appl. Math. Comput., 157, pp. 549-560

[17]. M. Sezer, A. Akyuz-Dascioglu, (2006). Taylor polynomial solutions of general linear differential-difference equations with 174, 753-765.

[18]. Pamuk, S. (2005), An Application for Linear and Nonlinear Heat Equations by Adomian's Decomposition Method, Appl. Math. Comput. 163, 89-96

[19]. Q. Wang, F. Fu, (2010) Solving Delay Differential Equations with Homotopy Analysis Method, Communications in variable coefficients, Appl. Math. Comput. 
Computer and Information Science, 97, 144-153.

[20]. R. Grzymkowski, D. Słota. (2005) Stefan problem solved by Adomian decomposition method. Int. J. Comput. Math., 82, pp. 851856

[21]. W. Al-Hayani, L. Casasus, (2005) The Adomian decomposition method in turning point problems, J. Comput. and Appl. Math. 177, 187-203.

[22]. W. Fushchych, R. Zhdanov, (1994) Antireduction and exact solutions of nonlinear heat equations, Nonlinear Math. Phys. 1 (1), 60-64.

[23]. X. Chen, L. Wang, (2010) The variational iteration method for solving a neutral functional-differential equation with proportional delays, Comput. Math. Appl., 59(8), 2696-2702.

[24]. Y. Cherruault, G. Adomian, K. Abbaoui, and R. Rach. (1995) Further remarks on convergence of decomposition method. International Journal of Bio-Medical Computing, 38:89-93.
[25]. Y.C. Jiao, Y. Yamamoto, C. Dang, and Y. Hao. (2002). An aftertreatment technique for improving the accuracy of Adomian's decomposition method. Computers and Mathematics with Applications, 43:783798.

[26]. Zel'dovich, Ya. B. and Kompaneets, A. S., (1995) On the theory of propagation of heat with the heat conductivity depending upon the temperature, pp. 61-71, In: Collection in Honor of the Seventieth Birthday of Academician A. F. Ioffe [in Russian], Izdat. Akad. Nauk SSSR, Moscow.

[27]. Z. Smarda, J. Dibl'ık, Y. Khan, (2005) Extension of the differential transformation method to nonlinear differential and integrodifferential equations with proportional delays, Advances in Difference Equations $69,1-13$. 